\title{
CircAPP Competes with APP mRNA to Promote Malignant Progression of Bladder Cancer
}

\section{Minjun Qi}

Affiliated No2 people's hospital of nanjing medical university

\section{Wei Cao}

Changzhou No 2 people's hospital

\section{Xingyu Wu}

changzhou no2 people's hospital

\section{Weijiang Mao}

changzhou no2 people's hospital

\section{Kai Cao}

wujin people's hospital

\section{Chao Lu}

changzhou no2 people's hospital

xiaowu liu ( $\square$ xiaowuliu1990@163.com )

wujin people's hospital https://orcid.org/0000-0003-4500-0023

\section{Li Zuo}

Changzhou no2 people's hospital

\section{Primary research}

Keywords: bladder cancer, malignant progression, miRNA-186, circAPP

Posted Date: April 12th, 2021

DOI: https://doi.org/10.21203/rs.3.rs-293144/v1

License: (c) (1) This work is licensed under a Creative Commons Attribution 4.0 International License.

Read Full License 


\section{Abstract}

Background: Bladder cancer $(\mathrm{BCa})$ is the most common cancer in the urinary system with high recurrence rate and poor prognosis. Circular RNA (circRNA) is a novel subclass of noncoding-RNA

which participate in progression of BCa. Here, we identified a novel circRNA-circAPP and aimed to investigate the role of circAPP in progression of $\mathrm{BCa}$.

Method: Public data of RNA sequencing was used to identify significant circRNA related to BCa. The role of circRNAs in progression in BCa was assessed in cytotoxicity assay, transwell assay and

flow cytometry. Biotin-coupled RNA pull-down and fluorescence in situ hybridization (FISH) were performed to evaluate the interaction between circRNAs and miRNAs.

Results: The expression of circAPP was higher in BCa tissues and cells than in normal samples. In vitro experiments showed that knockdown of circAPP inhibited cell proliferation and impeded the metastasis of BCa cells. Mechanistically, we demonstrated that circAPP acts as a sponge for miR-186-5p and promotes host gene APP's expression. Clinically, circAPP predicts worse overall survival of BCa patients, indicating its prognostic value.

Conclusion: Our study identified that circAPP modulates metastasis of BCa through miR-186-5p/APP aixs and may serve as a promising prognostic biomarker for $\mathrm{BCa}$, which provides novel insights into treatment of BCa.

\section{Introduction:}

Bladder cancer $(\mathrm{BCa})$ is the fourth most frequent cancer diagnosis in men and the most common malignant tumor of the urinary system. Bladder urothelial carcinoma (BUC) is the most pandemic subset of $\mathrm{BCa}$, accounting for approximately $90 \%$ of all cases of $\mathrm{BCa}$. Despite the development of treatment strategy for $\mathrm{BCa}$, the 5-year survival of $\mathrm{BCa}$ is still unsatisfied. Postoperative recurrence and distant metastasis make five-year prognosis for advanced $\mathrm{BCa}$ worse. In this regard, identifying novel biomarkers and potential therapeutic targets for $\mathrm{BCa}$ diagnosis and treatment, is urgently needed(1).

Circular RNAs (circRNAs) are a class of single stranded RNAs that constructs a closed loop by connecting the linear $5^{\prime}$ and $3^{\prime}$ ends(2). Due to its unique structure and stabilizing feature, circRNAs are considered to be promising biomarkers for prognosis and diagnosis of cancer patients, which draws growing attention(3). In regarding of $\mathrm{BCa}$, high-throughput sequencing and microarray identified a large number of novel dysregulated circRNAs in cell lines or tissues, indicating potential roles of these circRNAs in BCa development and progression $(4,5)$. Previous studies revealed that several oncogenic and antioncogenic circRNAs could regulate many aspects of malignant phenotype of BCa including cell proliferation, cell cycle arrest, apoptosis, metastasis, angiogenesis, and chemoresistance $(6,7)$. For example, CirclTCH inhibited cell proliferation by sponging miR-224 to up-regulated of PTEN in BCa(8). In addition, RAB27A 
promoted proliferation and chemoresistance of BCa by inducing protein transport and small GTPasemediated signal transduction. Despite of significant functions of circRNAs in $\mathrm{BCa}$, more work is needed to further identify novel circRNAs in prognosis and biomarker and explore their mechanisms in carcinogenesis(9).

In this study, we analyzed public RNA sequencing (RNA-Seq) and verified a upregulated circRNA-circAPP in $\mathrm{BCa}$ tissues. Functional experiments showed that circAPP expression promoted $\mathrm{BCa}$ invasion and proliferation. Furthermore, we found that circAPP modulated the expression of its host mRNA-APP by sponging miR-186, which activated metastasis of BCa. Finally, circAPP was identified to be a potential biomarker for prognosis of $\mathrm{BCa}$.

\section{Methods:}

Tissues and serum specimen collection This study has been approved by the Ethics Committee of the Nanjing Medical University Affiliated Cancer Hospital and was performed in accordance with the provisions of the Ethics Committee of Nanjing Medical University. We obtained the written informed consent from all the patients. 40 paired Human BCa tissues, ANT were obtained from the Department of Thoracic Surgery, Jiangsu Cancer Hospital between 2010 and 2016 (Nanjing, China).

Cell cultures The BIU-87 cells and 5637 cells were obtained from the Chinese Academy of Sciences Cell Bank and were authenticated by the providers by DNA-fingerprinting analysis or isoenzyme analysis, and were tested negative for mycoplasma contamination. BIU-87 cells and 5637 cells were cultured in RPMI1640 medium (Keygen Biotech, Nanjing, China) supplemented with $10 \%$ fetal bovine serum (Gibco, Grant Island, USA). Cells were maintained in an atmosphere of $5 \% \mathrm{CO} 2$ in a humidified $37^{\circ} \mathrm{C}$ incubator. Cells were authenticated by STR analysis at Guangzhou Cellcook Biotech Co., Ltd. (Guangzhou, China) Characterized Cell Line Core Facility within the last three years and routinely tested negative for mycoplasma contamination.

Over-expression or knockdown of genes Human circAPP linear sequence was obtained from esophageal squamous cell carcinoma tissues by PCR and inserted into plasmid vector pcDNA 3.1 (Hanbio, shanghai, China). Human APP cDNA was amplified with PCR primers and subcloned into pcDNA3.1 empty vector (Hanbio). The small interfering RNA (siRNA) of circAPP and mAPP were provided by RiboBio (Guangzhou, China). The transient transfection of the overexpressing plasmids were performed using the Lipofectamine 3000 kit (Invitrogen, Carlsbad, CA) according to manufacturer's instructions, and the transient transfection of siRNA were performed using the Lipofectamine iMax kit(Invitrogen) according to manufacturer's instructions.

RNase R treatment \& Quantitative PCR. Total RNA was isolated from cells and tissues using Trizol reagent (Life Technolohies, Scotland, UK) according to the manufacturer's protocol. And the RNA was extracted from serum with miRNeasy Mini Kit (Qiagen, Hilden,Germany). Nuclear and cytoplasmic RNA was extracted using nuclear and cytoplasmic RNA purification kit (Fisher scientific, Vilnius, Lithuania). For RNase R treatment, $1 \mu \mathrm{g}$ of total RNA was incubated 30 min at $37^{\circ} \mathrm{C}$ with or without $3 \mathrm{U}$ of RNase R 
(Geneseed, Guangzhou, China). Reverse transcription was then performed using random hexamers (Takara, Dalian, China) and quantitative PCR (qPCR) was performed using SYBR Green master mix (Applied biosystems, Vilnius, Lithuania). To quantify expression of circRNA transcripts, divergent primers were designed to amplify across the back-splicing junction. Amplification was performed using the StepOnePlus Real-Time PCR System (Applied Biosystems, Foster City, CA) and Ct thresholds were determined by the software. Expression was quantified using 2- $\triangle \triangle C T$ method using GAPDH (for mRNA/ circRNA) or U6 small nuclear RNA (for nuclear RNA fraction) as reference genes.

Western blotting Briefly, total protein of cells was extracted using RIPA (Thermo Fisher Scientific, Waltham, USA) with a cocktail of proteinase and phosphatase inhibitors (Thermo Fisher Scientific) according to its protocol. Equal amounts of protein lysates were resolved by SDS-PAGE gels and then transferred on a PVDF membrane (Millipore, Massachusetts, USA). After incubation with a primary antibody at $4^{\circ} \mathrm{C}$ overnight, the membranes were hybridized with a secondary antibody at room temperature for $1 \mathrm{~h}$. Blots were visualized using ECL detection (Thermo Fisher Scientific).

Transwell and Matrigel assay For migration assay, $4 \times 104$ cells were seeded into the upper transwell assay chambers with $8 \mu \mathrm{m}$ pore filters (Millipore) in serum-free medium. For invasion assay, $4 \times 104$ cells were seeded into the upper matrigel assay chambers with a matrigel-coated membrane (Corning, Massachusetts, USA) in serum-free medium. The lower chamber contained medium with $10 \% \mathrm{FBS}$ as chemokine. After incubation for 24 hours for migration and $48 \mathrm{~h}$ for invasion at $37^{\circ} \mathrm{C}$, non-migrating or non-invading cells were gently removed and cells migrated to the bottom of the membrane were fixed with $4 \%$ paraformaldehyde, stained with crystal violet solution for $30 \mathrm{~min}$, and visualized under a microscope at $\times 100$ magnification.

Wound-healing assay Transfected cells were cultured in 6-well plates. After the cells reached $90 \%$ confluence, a standard $200 \mu$ l pipette tip was subsequently utilized to scratch linear wounds. In addition, the cell monolayers were cultivated in FBS-free medium. After scratching, the images of the wound closure were captured at 0 , and $36 \mathrm{~h}$.

RNA pull-down The Biotin-labled RNA probes of circAPP and scramble were synthesized by GenePharma Company (Suzhou, China). RNA pull-down assay was performed using a Biotinylated Protein Interaction Pull-Down Kit (Thermo Fisher Scientific). In brief, 2×107 cells incubated in lysis buffer on ice for 30min. The streptavidin-coated magnetic beads were incubated with biotinylated probes at room temperature for $30 \mathrm{~min}$. The beads-probe complex was added to lysis, and mixed at $4^{\circ} \mathrm{C}$ for $2 \mathrm{~h}$. The bound miRNA were eluted from the packed beads. The miRNA in the capture complex were identified by qRT-PCR.

RNA-Fluorescence in situ hybridization assay and Fluorescence immunocytochemical staining RNAFluorescence in situ hybridization (FISH) assays were performed using a RNA-FISH kit (GenePharma, China) according to the manufacturer's instructions. Cy3-labeled antisense probe was synthesized by GenePharma company (Suzhou, China) against the junction site of circAPP. In briefly, 5637 cells were fixed with $4 \%$ paraformaldehyde. After pre-hybridization with $1 \times$ PBS $/ 0.5 \%$ Triton X-100, cells were 
blocked and hybridized in hybridization buffer with Cy3-labeled probe at $37^{\circ} \mathrm{C}$ overnight. Cells were stained with DAPI (300 nmol/L).

Statistics All statistical analyses were performed with SPSS 25.0 software. Qualitative variables were analyzed by chi-square test or fisher's exact test. For continuous variables, if which obey the normal distribution, student's $t$ test is used to compare the differences. Otherwise, variables were compared using nonparametric test for which with an abnormal distribution. Differences between groups were compared using analysis of variance (ANOVA) when applicable or a nonparametric test. Correlation analysis was performed using the Pearson correlation coefficient method. Unless otherwise specified, the results are presented as the means \pm standard deviation (SD). All statistical tests were 2 sided, and $P<0.05$ was considered statistically significant.

\section{Data availability}

The circRNA microarray is available in the Gene Expression Omnibus (GEO) (https://www.ncbi.nlm.nih.gov/geo/query/acc.cgi) under accession numbers GSE147985). The source data of other figures are provided as a Source Data file. All other data are available from the authors upon reasonable requests.

\section{Results:}

\section{Identification and characterization of circAPP in bladder cancer.}

We firstly analyzed the published circRNA microarray data of human bladder cancer (BCa) tissues and paired normal bladder tissues(1). And we found circAPP was significantly upregulated in BCa (Fig. 1A). CircAPP (circBase(2) ID: hsa_circ_0003323) was derived from the 12 and 13 exons of Amyloid Beta Precursor Protein (APP) gene (Fig. 1B). CircAPP was significantly increased in 40 paired BCa tissues compared to normal tissues (Fig. 1C). We confirmed that circAPP was relative enriched in 5637 BCa cell line and less enriched in BIU-87 BCa cell line compared with the normal urothelial cell line SV-HUC-1 (Fig. 1D). CircAPP was only y detectable in cDNA but not genomic DNA (gDNA) from 5637 cell lines by qRT-PCR with divergent primers, while mAPP could be amplified in both cDNA and gDNA using convergent primers (Fig. 1E). Besides, RNase R digestion assay showed that the circular isoform was resistant to RNase $\mathrm{R}$, whereas the linear isoform was obviously decreased after RNase R treatment (Fig. 1F). In addition, the subcellular localization of circAPP was detected with qRT-PCR analysis using nuclear and cytoplasmic fractions of 5637 cells and FISH assay. We found that circAPP was enriched in the cytoplasm fraction and mainly distributed in the cytoplasm (Fig. 1G). Taken together, these results indicated that circAPP was up-regulated in BCa tissues and cell lines and was predominantly localized in the cytoplasm.

\section{CircAPP promoted the invasion and migration of Bca cells.}


To investigate the potential biological effect of circAPP on BCa cells, we established circAPP stably overexpressing BIU-87 cell lines via transfecting with circAPP vector. We also used RNA interference (siRNA) to silence the expression of circAPP in 5637 cells. The overexpression and knock-down efficiencies of circAPP, mAPP, and PreAPP were detected by qRT-PCR analysis, surprisingly, we found that the circAPP and mAPP were upregulated upon overexpressed plasmid transfected. And circAPP and mAPP were decrease after siRNA was transfected (Fig. 2A-B). The results of transwell, matri gel, and wound healing demonstrated that circAPP facilitated the aggressive the BCa cells (Fig. 2C-E).

\section{CircAPP upregulated mAPP via competing sponge endogenous miR-186-5p.}

To explore the mechanism of circAPP regulated mRNA of its host gene, we analyzed the mAPP-CLIP seq data in StarBase database(3), there were 153 miRNA recognized could interact with the 3' UTR region of mAPP. There were nine miRNA could been potentially sponged by circAPP in StarBase with tight screening criteria. With comprehensively analyzing the two data, there were six miRNAs in common (Fig. 3A). After screened the expressed correlation between candidate miRNAs and mAPP, we found that only miR-186-5p was significantly negative related to the expression of mAPP (Fig. 3B). The positions of putative binding sites in circAPP and mAPP were analyzed in StarBase (Fig. 3C). To explore whether circAPP and mAPP can act as effective miRNA sponges, we performed Argonaut 2 (Ago2) reciprocal immunoprecipitation (RIP) assay. The results demonstrated that both circAPP and mAPP could efficiently adsorbed Ago2 protein (Fig. 3D). The biotinylated circAPP probe, mAPP probe, and scramble probe were designed and applied to perform RNA pull-down assay. The pull-down efficiency was verified in 5637 via qRT-PCR. The results released that both circAPP and mAPP could pulled down miR-186-5p (Fig. 3E-F). The biotin-coupled miR-186-5p were used to confirm the interaction (Fig. 3G). To confirm the sponge effect between circAPP/mAPP and miR-186-5p, a dual- luciferase reporter assay indicated that transfection of wildtype or mutation circAPP/mAPP reporter plasmids, the results released that wildtype markedly attenuated the luciferase activity, and verified that circAPP/mAPP could bind with miR-186-5p (Fig. 4A-B). Futhermore, Dual RNA-FISH assay confirmed the colocalization of circAPP/mAPP and miR186-5p (Fig. 4C-D). The RNA pull-down assay demonstrated that circAPP sponged more miR-186-5p upon circAPP overexpressed (Fig. 4E). We constructed the mAPP overexpression plasmid (Fig. 4F), circAPP harbor less miR-186-5p after mAPP overexpressing (Fig. 4G). A miR-186-5p inhibitor was designed to decrease the expression of miR-186-5p (Fig. 4H). Moreover, transfection of miR-186-5p mimic abrogated the effects of circAPP on promoting mAPP increased, and miR-186-5p inhibitor abolished the effects of circAPP knockdown on suppressing the expression of mAPP (Fig. 4I-J). Taken togther, we these results indicated that circAPP regulated the expression of mAPP via competing endogenous miR-186-5p.

\section{The expression of circAPP was positive related to the expression of mAPP.}

We firstly applied qRT-PCR to detect the correlations between circAPP and mAPP in $40 \mathrm{BCa}$ tissues and matched adjacent normal tissues. We found that circAPP was significant positive related to mAPP (Fig. 5A). As the same as circAPP, mAPP was upregulated in BCa tissues (Fig. 5B). In TCGA database, the Kaplan-Meier survival curves released that BCa patients with higher miR-186-5p expression level had a 
better overall survival (OS) (Fig. 5C). BCa patients with higher mAPP expression level had a worse prognosis (Fig. 5D). In BCa tissues, the results of In Situ Hybridization (ISH) assay released that the expression of APP positively associated with the expression of circAPP (Fig. 5E). Taken togther, these findings suggested that circAPP regulated the MAPP in BCa and could as a potential diagnosis and therapy biomarker for $\mathrm{BCa}$.

In conclusion, we identified a novel oncogenic player circAPP from the BCa circRNA microarray, and verified the results using 40 paired $\mathrm{BCa}$ tissues and $\mathrm{BCa}$ cell lines. We released that circAPP promoted the metastasis of BCa cells. Importantly, circAPP could increase the expression of its host gene APP via competing endogenous miR-186-5p. Therefore, circAPP may be a promising independent prognostic biomarker and potential target in BCa therapy (Fig. 6).

\section{Discussion:}

In this study, we explored the effect of circAPP on the metastasis of $\mathrm{BCa}$ and demonstrate the regulatory mechanism of miR-186-5p/APP positive feed-back loop pathway. We first discovered that circAPP is frequently upregulated in $\mathrm{BCa}$ and correlated with poor patient prognosis, indicating its applicability as a promising prognostic biomarker in $\mathrm{BCa}$. In addition, we demonstrated that the inhibition of circAPP reversed the metastasis of $\mathrm{BCa}$ cells and thus inhibited the progression of $\mathrm{BCa}$. Furthermore, we revealed that circAPP acted as a positive feed-back loop and regulated the expression of APP via miR-186-5p. These results suggested that circAPP might promote the progression of $\mathrm{BCa}$.

At early time, circRNAs were defined as a type of circular RNA transcript via aberrant RNA splicing and initially regarded as functionless byproducts(1). However, circRNA's functions in cancer have been increasingly reported with the rapid spread of high-throughput sequencing(10). Based on published studies, circRNA could involved in various pathological processes via miRNA sponges, interacting with RNA binding proteins, transcription or splicing, and translating proteins $(11-13)$. The role of circRNAs and the underlying mechanisms in $\mathrm{BCa}$ has been reported before. However, more specific mechanisms of circRNAs in metastasis of BCa need to be further identified. Using published circRNA microarray data of human bladder cancer (BCa), we identified an up-regulated circRNA-circAPP (circBase(2) ID: hsa_circ_0003323), which was derived from the 12 and 13 exons of Amyloid Beta Precursor Protein (APP) gene $(14,15)$. Most circRNAs are generally recognized to be low expression in tumor, probably due to RNA splicing process affected by accelerating cellular proliferation rate(16). But high-throughput sequencing technology identify several circRNAs enriched in tumor tissues. In order to confirm the trend, we validated the expression of circAPP in 40 paired tumor and normal tissues, which was consistent with sequencing's result. In addition, loss-of-function experiments revealed that knockdown of circAPP inhibited the metastasis of BCa cells in vitro, which indicated that circAPP might play a vital role in progression of $\mathrm{BCa}$.

The host gene of circAPP is Amyloid precursor protein (APP) which is a transmembrane precursor protein and is widely expressed in the central nervous system and peripheral tissues including the liver and 
bladder $(15,17)$. Published studies revealed that APP usually cleaved and produced a variety of short peptides, which exerts different physiological properties and functions in metabolic disease and cancers. Tsang et al found APP cleaved and generated SAPPa mediating breast cancer migration and proliferation(18). In addition, they reported that patients with positive APP expression may require vigilant monitoring of their disease and more aggressive therapy in another study(19). Furthermore, zhang et al revealed that APP was significantly increased in the human bladder cancer tissues compared with matched normal bladder tissues and inhibited proliferation, migration and invasion of human bladder cancer cells(20). Mechanically, knockdown of APP significantly decreased the phosphorylation of extracellular regulated protein kinases(20). Obviously, APP is an oncogene in BCa. Intriguingly, circRNA could regulate or facilitate the function of host gene in disease progression via multi-ways. For example, su et al uncover circPHIP enhances its malignancy via miR-142-5p which directly targets the expression of PHIP and ACTN4(4). In addition, SMO-193a.a, encoded by circSMO, induced SMO activation via interacting with SMO, enhancing SMO cholesterol modification, and releasing SMO from the inhibition of patched transmembrane receptors(3). Those results indicated a potential relationship between circRNAs and host genes, which may one of the significant functions of circRNAs. In order to explore the relationship between circAPP and APP, we analyzed the expression correlation and identified a positive correlation between them, indicating a potential regulated role. Mechanically, sponging miRNAs is one of the most common and significant role of circRNAs regulating the progression of cancer, which constructing a competition relationship between circRNAs with targeted mRNAs. Previous studies identified that most circRNAs have miRNA-binding sites. Since then, miRNA sponge function of circRNAs has been comprehensively investigated in many biological processes(10). Li et al revealed that circARNT2 functions as an oncogene by sponging miR-155-5p, leading to PDK1 upregulation, and finally sensitizes HCC cells to cisplatin(7). We primarily found six miRNAs in common via overlapping mAPPCLIP seq data with circRNA binding miRNAdata in StarBase database with tight screening criteria(21). After screened the expressed correlation between candidate miRNAs and mAPP, we found that only miR186-5p was significantly negative related to the expression of mAPP. In addition, biotinylated RNA pulldown assay and an RNA FISH assay further validated and confirmed the interaction between circAPP and miR-186-5p in BCa. Furthermore, the expression of circAPP was significant inverse correlated with the expression of miR-186-5p in BCa tissues. Therefore, circAPP might serve as a sponge for miR-186-5p and thus perform a series of functions.

Due to highly conserved and broadly expression, covalently closed loop structures and stability, and tissue-specific features, previous investigations indicate that circRNAs demonstrate promising and considerable potential for use as diagnostic and prognostic biomarkers in cancers $(8,16)$. Among BCa, apart from tissues detection, circRNAs can be also detected in blood and urine. Here, we detected and revealed an up-regulated trend of circAPP in cancer patients' blood compared with those of health. Also, circAPP can discriminate the poor survival patients from BCa patients, indicating the prongostic function in clinical, while further exploration is clearly warranted in other external cohorts.

However, there are still several limitations in this study. Firstly, we didn't perform the functions of circAPP in vivo experiments. Further investigation need to be completed. At the same time, the upstream 
regulatory mechanism of circAPP were not investigated. Furthermore, the mechanism of circAPP regulating the progression of $\mathrm{BCa}$ is not comprehensively explored. Perhaps circAPP could also exerts its functions via RNA binding proteins, transcription or splicing, and translating proteins ways. These issues should be further pursued in subsequent studies.

\section{Conclusion:}

In summary, our research indicates that circAPP is highly expressed in BCa tissues and acts as oncogenes in development and progression of BCa. Mechanistically, circAPP promotes metastasis of BCa by adsorbing miR-186-5p and in turn increasing APP expression. This circAPP/miR-186-5p/APP axis provides novel insights and strategies for $\mathrm{BCa}$.

\section{Declarations}

\section{Ethics approval and consent to participate:}

The study was approved by the Regional Ethics Committee at The Afliated Changzhou No. 2 People's Hospital of Nanjing Medical University.

\section{Consent for publication:}

The consent was obtained from patients.

\section{Availability of data and materials:}

Yes.

\section{Acknowledgements \& Funding}

This work was supported by the Science and Technology supporting plan of Changzhou (WS201809, WZ201931).

\section{Statement of contribution}

MJQ, XWL, and LZ designed the study and performed major experiments. WC and XYW supported the study. WJM, KC, and CL are in charge of collecting the information of patients. WJM, CL, and LZ performed the bioinformatics analysis and statistics analysis. WC and MJQ helped in writing the draft.

\section{Conflict of interest (Competing interests):}

The authors declared no conflicts of interest in this work.

\section{References}


1. Yang $X, Y e T$, Liu H, Lv P, Duan C, Wu X, et al. Expression profiles, biological functions and clinical significance of circRNAs in bladder cancer. (1476-4598 (Electronic)).

2. Tsitsipatis D, Grammatikakis I, Driscoll RK, Yang X, Abdelmohsen K, Harris SC, et al. AUF1 ligand circPCNX reduces cell proliferation by competing with p21 mRNA to increase p21 production. (13624962 (Electronic)).

3. Wu X, Xiao S, Zhang M, Yang L, Zhong J, Li B, et al. A novel protein encoded by circular SMO RNA is essential for Hedgehog signaling activation and glioblastoma tumorigenicity. (1474-760X (Electronic)).

4. Su W, Shen $Y$, Wang $Y$, Wang F, Hong $X$, Chen $Y$, et al. circPHIP promotes oral squamous cell carcinoma progression by sponging miR-142-5p and regulating PHIP and ACTN4 expression. (21622531 (Print)).

5. Jin M, Lu S, Wu Y, Yang C, Shi C, Wang Y, et al. Hsa_circ_0001944 promotes the growth and metastasis in bladder cancer cells by acting as a competitive endogenous RNA for miR-548. (17569966 (Electronic)).

6. Zeng W, Liu Y, Li WT, Li Y, Zhu JA-O. CircFNDC3B sequestrates miR-937-5p to derepress TIMP3 and inhibit colorectal cancer progression. (1878-0261 (Electronic)).

7. Li Y, Zhang Y, Zhang S, Huang D, Li B, Liang G, et al. circRNA circARNT2 Suppressed the Sensitivity of Hepatocellular Carcinoma Cells to Cisplatin by Targeting the miR-155-5p/PDK1 Axis. (2162-2531 (Print)).

8. Yang C, Yuan W, Yang X, Li P, Wang J, Han J, et al. Circular RNA circ-ITCH inhibits bladder cancer progression by sponging miR-17/miR-224 and regulating p21, PTEN expression. (1476-4598 (Electronic)).

9. Huang X, Li Z, Zhang Q, Wang W, Li B, Wang L, et al. Circular RNA AKT3 upregulates PIK3R1 to enhance cisplatin resistance in gastric cancer via miR-198 suppression. (1476-4598 (Electronic)).

10. Yang C, Mou Z, Zhang Z, Wu S, Zhou Q, Chen Y, et al. Circular RNA RBPMS inhibits bladder cancer progression via miR-330-3p/RAl2 regulation. (2162-2531 (Print)).

11. Tan S, Kang Y, Li H, He HQ, Zheng L, Wu SQ, et al. circST6GALNAC6 suppresses bladder cancer metastasis by sponging miR-200a-3p to modulate the STMN1/EMT axis. (2041-4889 (Electronic)).

12. Liang Z, Guo W, Fang S, Zhang Y, Lu L, Xu W, et al. CircRNAs: Emerging Bladder Cancer Biomarkers and Targets. (2234-943X (Print)).

13. Okholm TA-O, Sathe S, Park SS, Kamstrup AB, Rasmussen AM, Shankar A, et al. Transcriptome-wide profiles of circular RNA and RNA-binding protein interactions reveal effects on circular RNA biogenesis and cancer pathway expression. (1756-994X (Electronic)).

14. Sliker BH, Goetz BT, Peters HL, Poelaert BJ, Borgstahl GEO, Solheim JC. Beta 2-microglobulin regulates amyloid precursor-like protein 2 expression and the migration of pancreatic cancer cells. (1555-8576 (Electronic)).

15. Liu D, Zha L, Liu Y, Zhao X, Xu X, Liu S, et al. $\beta 2$-AR activation promotes cleavage and nuclear translocation of Her2 and metastatic potential of cancer cells. (1349-7006 (Electronic)). 
16. Xie F, Xiao X, Tao D, Huang C, Wang L, Liu F, et al. circNR3C1 Suppresses Bladder Cancer Progression through Acting as an Endogenous Blocker of BRD4/C-myc Complex. (2162-2531 (Print)).

17. Liang TA-O, Sang S, Shao Q, Chen C, Deng Z, Wang T, et al. Abnormal expression and prognostic significance of EPB41L1 in kidney renal clear cell carcinoma based on data mining. (1475-2867 (Print)).

18. Tsang JYS, Lee MA, Chan TH, Li J, Ni YB, Shao Y, et al. Proteolytic cleavage of amyloid precursor protein by ADAM10 mediates proliferation and migration in breast cancer. (2352-3964 (Electronic)).

19. Tsang JYS, Lee MA, Ni YB, Chan SK, Cheung SY, Chan WW, et al. Amyloid Precursor Protein Is Associated with Aggressive Behavior in Nonluminal Breast Cancers. (1549-490X (Electronic)).

20. Zhang D, Zhou C, Li Y, Gao L, Pang Z, Yin G, et al. Amyloid precursor protein is overexpressed in bladder cancer and contributes to the malignant bladder cancer cell behaviors. (1442-2042 (Electronic)).

21. Bi J, Liu H, Dong W, Xie W, He Q, Cai Z, et al. Correction to: Circular RNA circ-ZKSCAN1 inhibits bladder cancer progression through miR-1178-3p/p21 axis and acts as a prognostic factor of recurrence. (1476-4598 (Electronic)).

\section{Tables}

Primers, Probes, and RNA sequences used in ths study

\begin{tabular}{|ll|}
\hline \multicolumn{2}{|l|}{ Primers for qRT-PCR } \\
\hline Primer Name & Sequence $\left(5^{\prime}{ }^{\prime}{ }^{\prime}\right)$ \\
\hline circAPP-F & TCAGTCTCTCTCCCTGCTCT \\
\hline circAPP-R & TGTGCTGTCTGTCCTTCTGT \\
\hline mAPP-F & CAAGCAGTGCAAGACCCATC \\
\hline mAPP-R & AGAAGGGCATCACTTACAAACTC \\
\hline preAPP-F & AGTCCCACTCCATCAACCAG \\
\hline preAPP-F & ATCTGAGAATGGTGTGGGCA \\
\hline miR-185-5p & UGGAGAGAAAGGCAGUUCCUGA \\
\hline GAPDH-F & GGAGCGAGATCCCTCCAAAAT \\
\hline GAPDH-R & GGCTGTTGTCATACTTCTCATGG \\
\hline U1-F & ATTGTGGCATTGCGCCGTA \\
\hline U1-R & CGCTCAATCTTTTCCCGTCTTT \\
\hline
\end{tabular}


FISH probe

circAPP $\quad$ ''-су3-TCAGGATGAAGTTGCCTCGTCACG-5'

mAPP 3'-FAM-TCCGGTGTAGCTGCCGCACGTAGCAAAGGCGAAGATGGCAAAGAC-5'

Pull-down probe

circAPP 3'-biotin-TCAGGATGAAGTTGCCTCGTCACG-5'

mAPP 3'-biotin-TCCGGTGTAGCTGCCGCACGTAGCAAAGGCGAAGATGGCAAAGAC-5'

scramble $\quad 3^{\prime}$-biotin-GTGTAACACGTCTATACGCCCA-5'

\begin{tabular}{|ll|}
\hline siRNAs & \\
\hline Oligo set & Target sequences \\
\hline si-circAPP\#1 & TGAAGTTGCCTCGTCACGT \\
\hline si-circAPP\#2 & AGGATGAAGTTGCCTC \\
\hline
\end{tabular}

\begin{tabular}{|l|l|}
\hline \multicolumn{2}{|l|}{ hsa-miR-185-5p mimics and inhibitors ( sense sequence) } \\
\hline mimics NC & UUCUCCGAACGUGUCACGUTT \\
\hline inhibitor NC & CAGUACUUUUGUGUAGUACAA \\
\hline hsa-miR-185-5p mimic & UGGAGAGAAAGGCAGUUCCUGA \\
\hline hsa-miR-185-5p inhibitor & ACCTCTCTTTCCGTCAAGGACT \\
\hline
\end{tabular}

The antibodies used in this study

\begin{tabular}{llll} 
Antibody & Supplier & Catalogue number & Host \\
\hline APP & abcam & ab241592 & R \\
GAPDH & abcam & ab8245 & R \\
\hline
\end{tabular}

Figures 
A

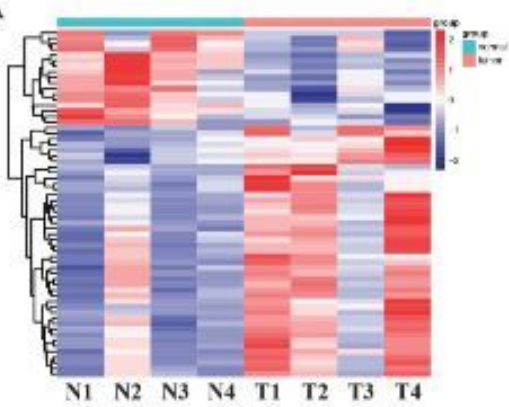

C

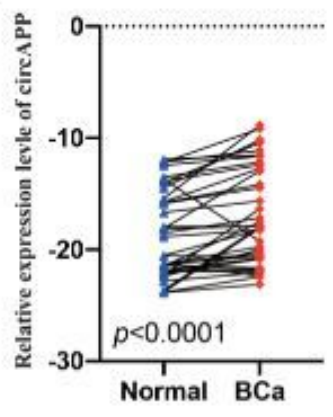

E

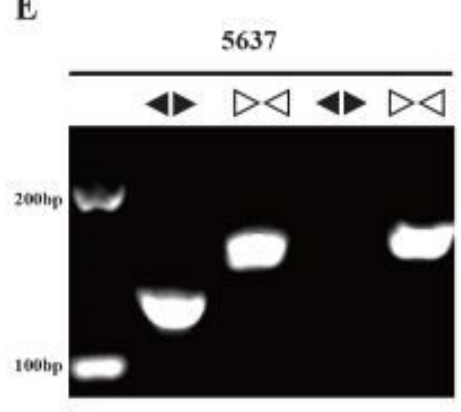

G

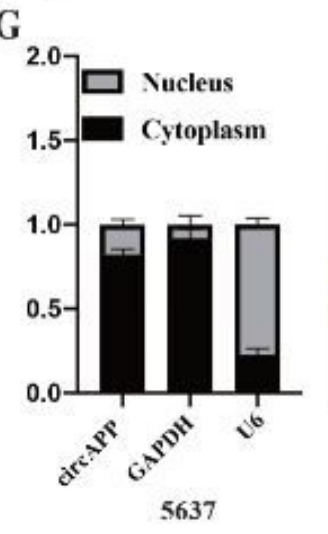

B

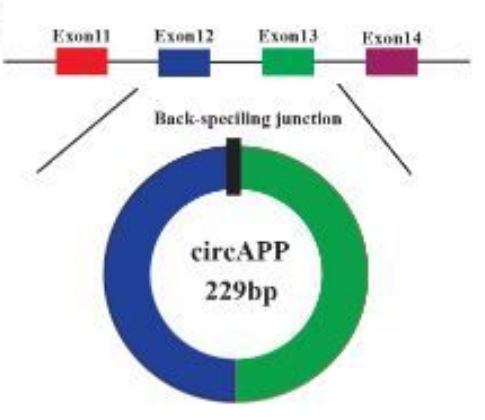

D

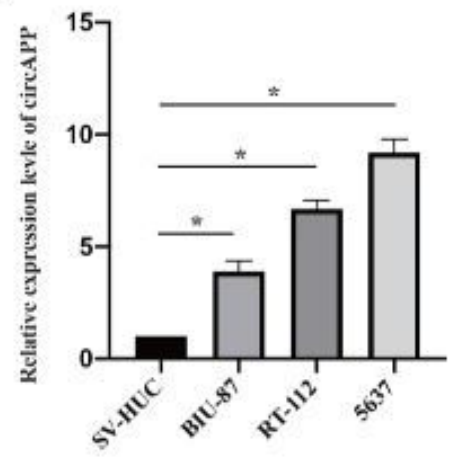

F

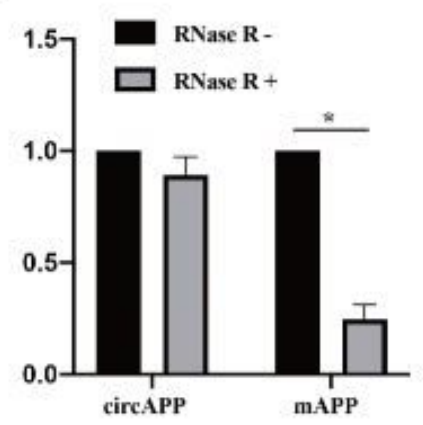

\section{Figure 1}

We firstly analyzed the published circRNA microarray data of human bladder cancer (BCa) tissues and paired normal bladder tissues(1). And we found circAPP was significantly upregulated in BCa (Fig. 1A). CircAPP (circBase(2) ID: hsa_circ_0003323) was derived from the 12 and 13 exons of Amyloid Beta Precursor Protein (APP) gene (Fig. 1B). CircAPP was significantly increased in 40 paired BCa tissues compared to normal tissues (Fig. 1C). We confirmed that circAPP was relative enriched in 5637 BCa cell 
line and less enriched in BIU-87 BCa cell line compared with the normal urothelial cell line SV-HUC-1 (Fig. 1D). CircAPP was only y detectable in cDNA but not genomic DNA (gDNA) from 5637 cell lines by qRTPCR with divergent primers, while mAPP could be amplified in both CDNA and gDNA using convergent primers (Fig. 1E). Besides, RNase R digestion assay showed that the circular isoform was resistant to RNase R, whereas the linear isoform was obviously decreased after RNase R treatment (Fig. 1F). In addition, the subcellular localization of circAPP was detected with qRT-PCR analysis using nuclear and cytoplasmic fractions of 5637 cells and FISH assay. We found that circAPP was enriched in the cytoplasm fraction and mainly distributed in the cytoplasm (Fig. 1G).

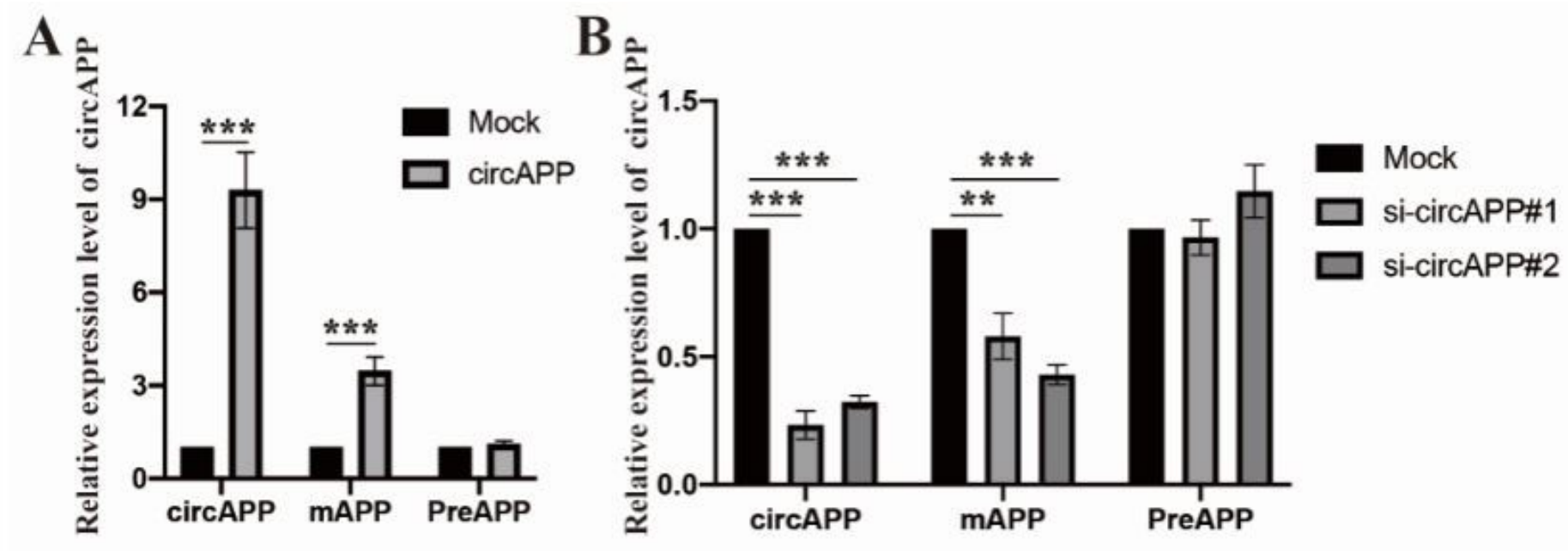

C
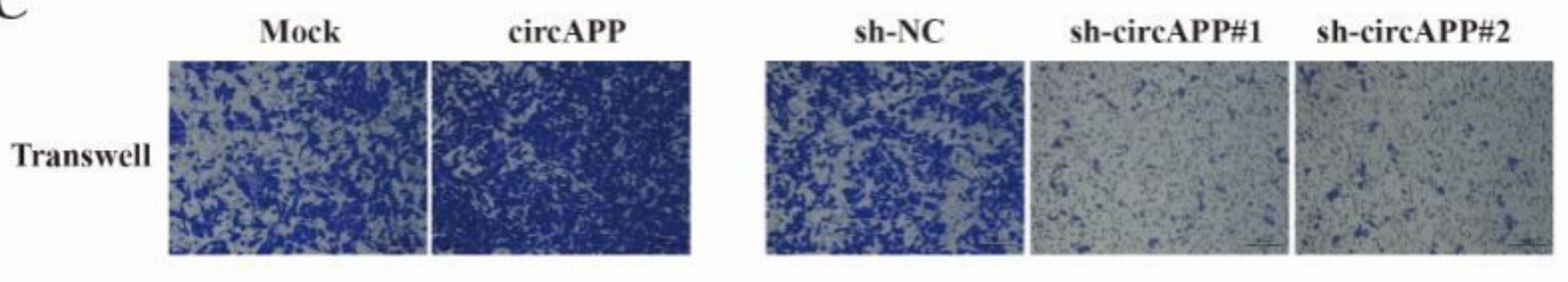

D

Matrigel
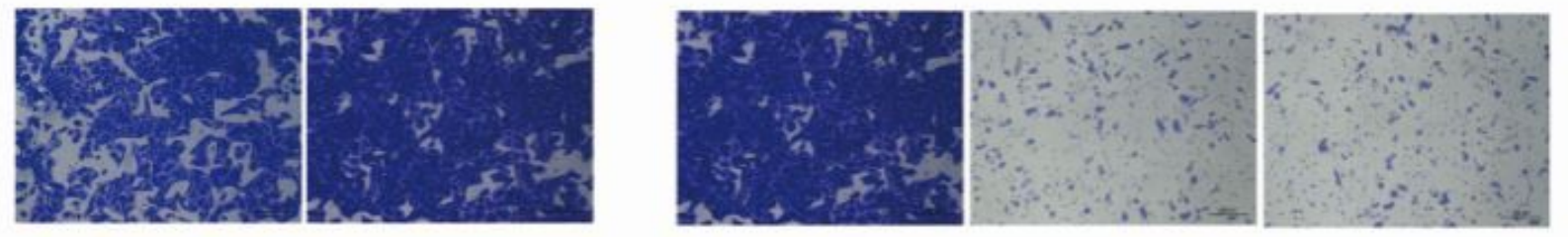

E
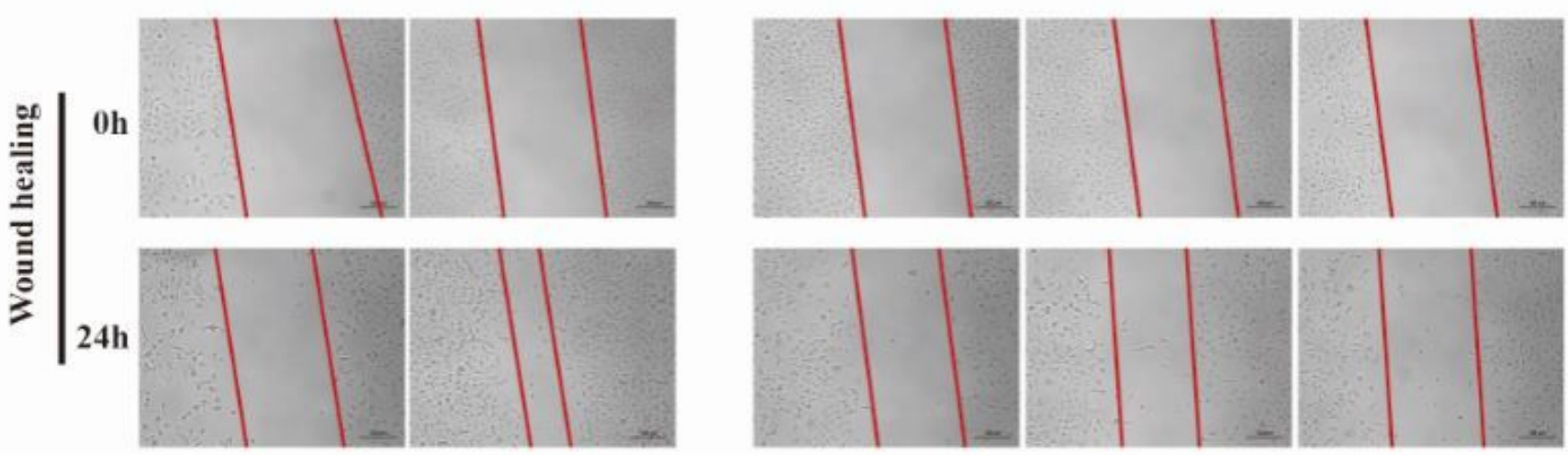
Figure 2

We also used RNA interference (siRNA) to silence the expression of circAPP in 5637 cells. The overexpression and knock-down efficiencies of circAPP, mAPP, and PreAPP were detected by qRT-PCR analysis, surprisingly, we found that the circAPP and mAPP were upregulated upon overexpressed plasmid transfected. And circAPP and mAPP were decrease after siRNA was transfected (Fig. 2A-B). The results of transwell, matri gel, and wound healing demonstrated that circAPP facilitated the aggressive the BCa cells (Fig. 2C-E).

A

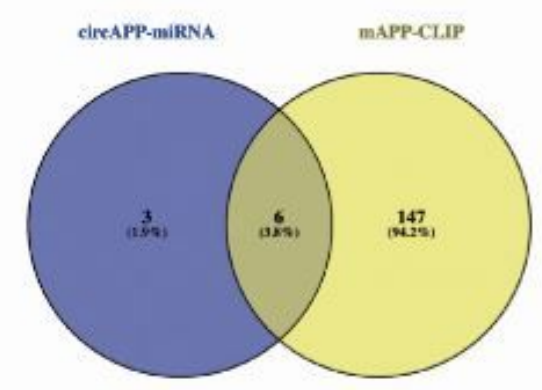

StarBase Database

C circAPP-miR-185-5p bind site Target: 5' ucuccagcceuggcauucuUuu $3^{\text {. }}$ $:|1|:|=1|||||||$ miRaA : $3^{\circ}$ uegGoUUUUCCUCUUARGMARe $5^{*}$
B

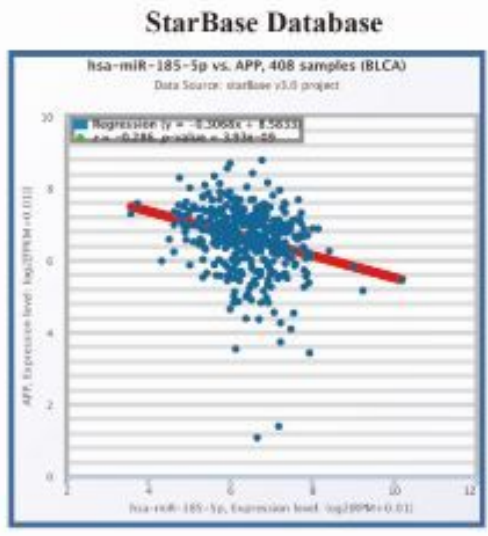

mAPP-miR-185-5p bind site

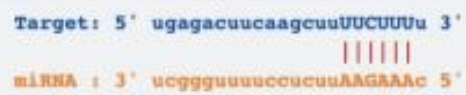

E

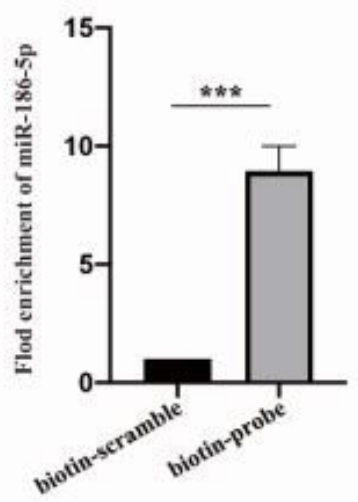

G
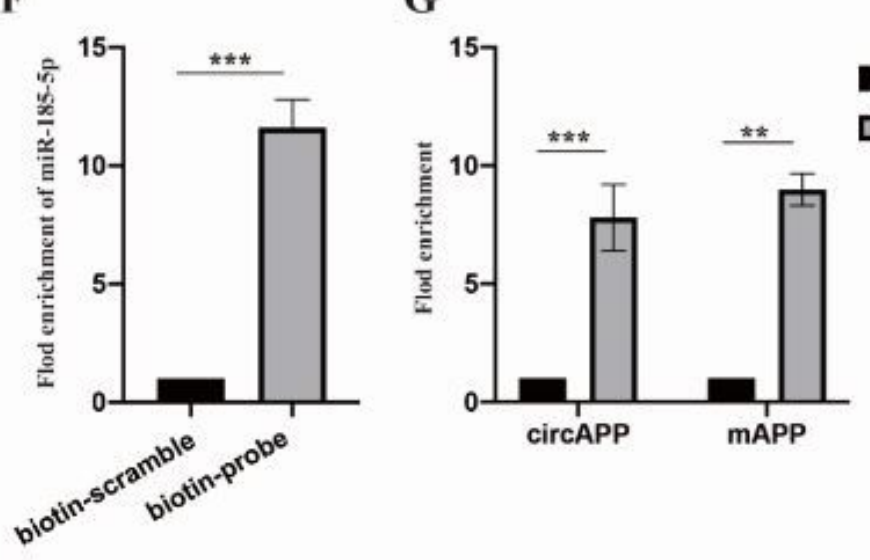


\section{Figure 3}

There were nine miRNA could been potentially sponged by circAPP in StarBase with tight screening criteria. With comprehensively analyzing the two data, there were six miRNAs in common (Fig. 3A). After screened the expressed correlation between candidate miRNAs and mAPP, we found that only miR-186-5p was significantly negative related to the expression of mAPP (Fig. 3B). The positions of putative binding sites in circAPP and mAPP were analyzed in StarBase (Fig. 3C). To explore whether circAPP and mAPP can act as effective miRNA sponges, we performed Argonaut 2 (Ago2) reciprocal immunoprecipitation (RIP) assay. The results demonstrated that both circAPP and mAPP could efficiently adsorbed Ago2 protein (Fig. 3D). The biotinylated circAPP probe, mAPP probe, and scramble probe were designed and applied to perform RNA pull-down assay. The pull-down efficiency was verified in 5637 via qRT-PCR. The results released that both circAPP and mAPP could pulled down miR-186-5p (Fig. 3E-F). The biotincoupled miR-186-5p were used to confirm the interaction (Fig. 3G). 
A

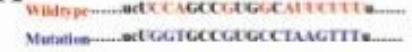

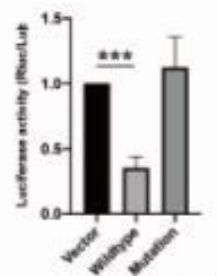

C
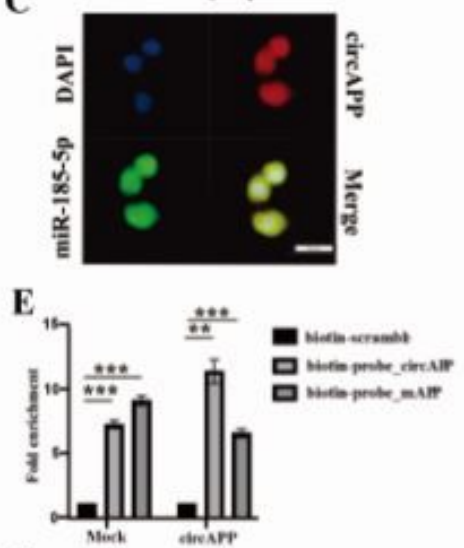

G

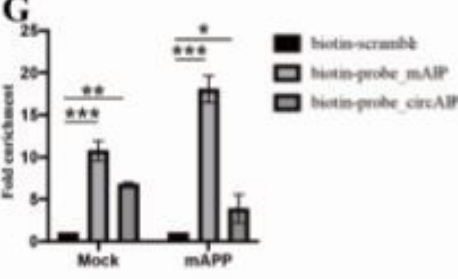

I

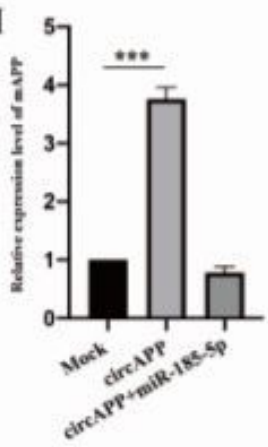

APP

GAPDH
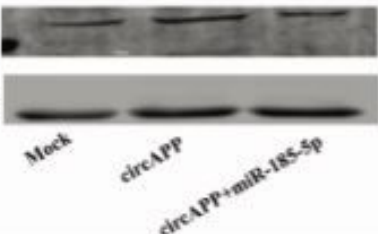

B

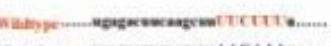

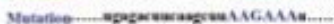

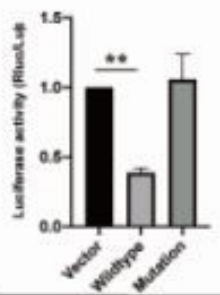

D
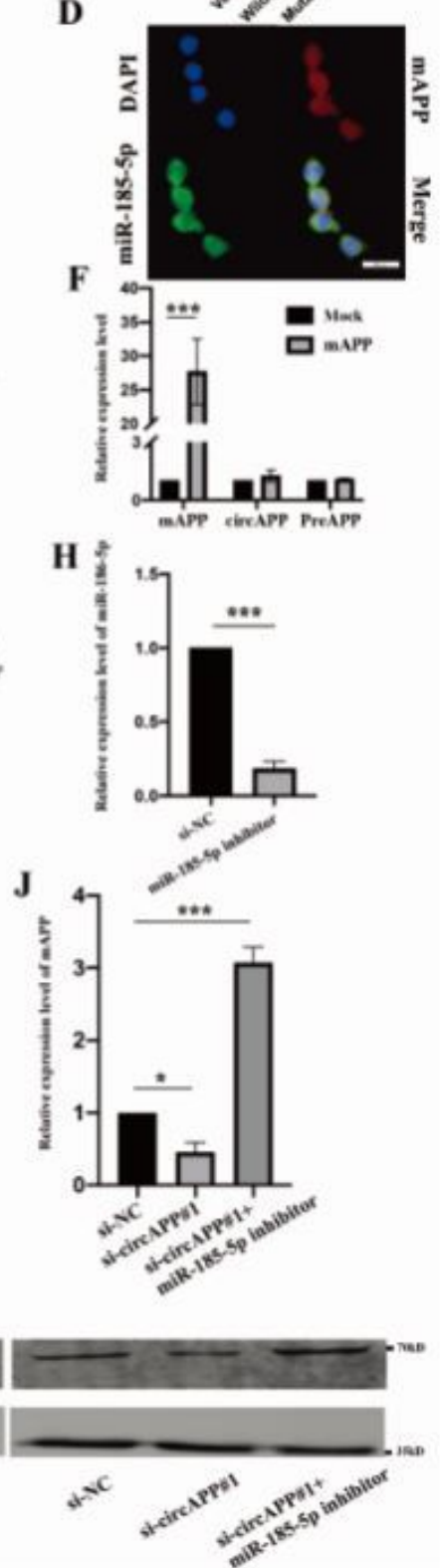

Figure 4

To confirm the sponge effect between circAPP/mAPP and miR-186-5p, a dual- luciferase reporter assay indicated that transfection of wildtype or mutation circAPP/mAPP reporter plasmids, the results released that wildtype markedly attenuated the luciferase activity, and verified that circAPP/mAPP could bind with miR-186-5p (Fig. 4A-B). Futhermore, Dual RNA-FISH assay confirmed the colocalization of circAPP/mAPP and miR-186-5p (Fig. 4C-D). The RNA pull-down assay demonstrated that circAPP sponged more miR- 
186-5p upon circAPP overexpressed (Fig. 4E). We constructed the mAPP overexpression plasmid (Fig. 4F), circAPP harbor less miR-186-5p after mAPP overexpressing (Fig. 4G). A miR-186-5p inhibitor was designed to decrease the expression of miR-186-5p (Fig. 4H). Moreover, transfection of miR-186-5p mimic abrogated the effects of circAPP on promoting mAPP increased, and miR-186-5p inhibitor abolished the effects of circAPP knockdown on suppressing the expression of mAPP (Fig. 4I-J).
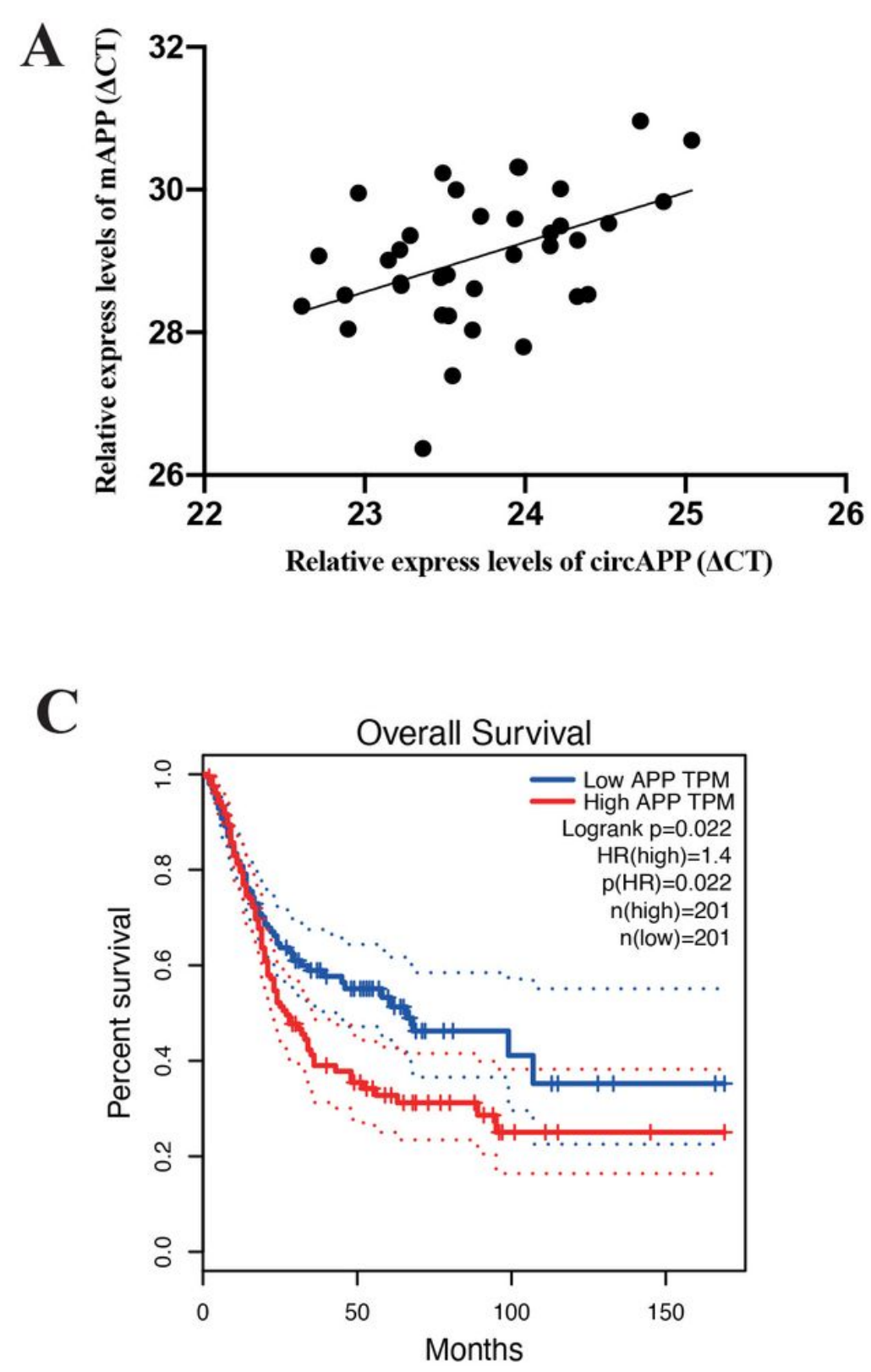

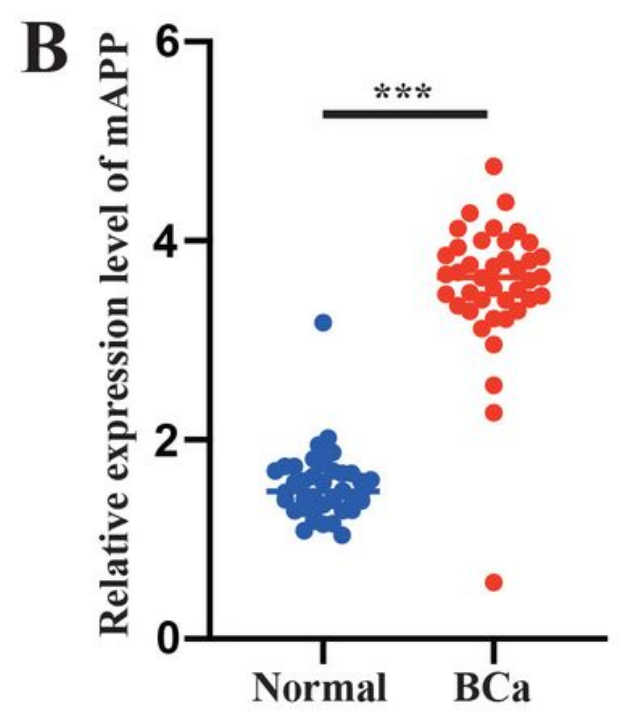

D

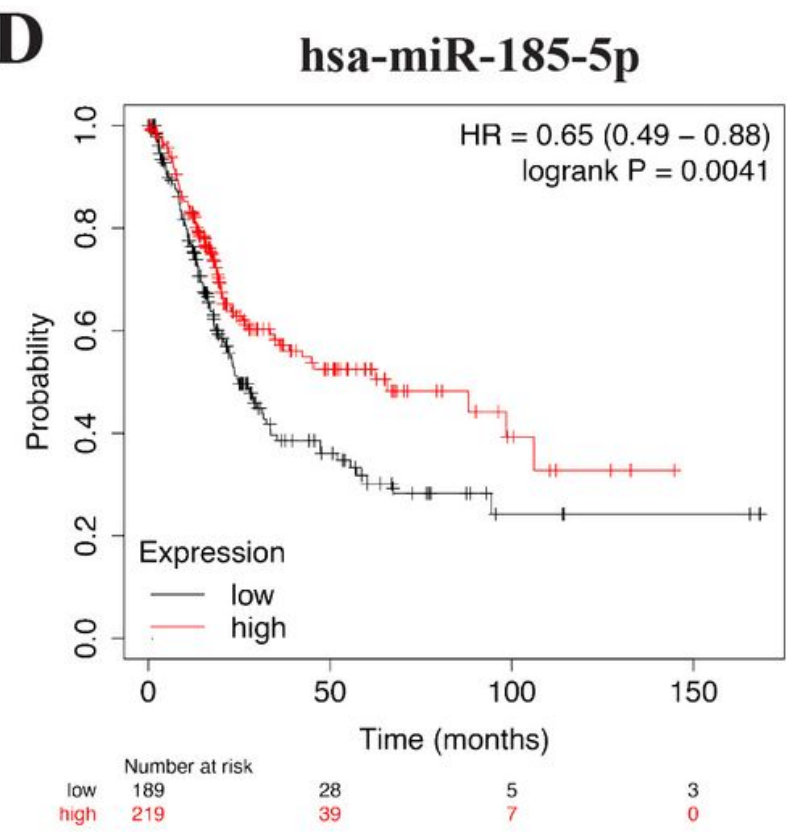

Figure 5

We found that circAPP was significant positive related to mAPP (Fig. 5A). As the same as circAPP, mAPP was upregulated in BCa tissues (Fig. 5B). In TCGA database, the Kaplan-Meier survival curves released that BCa patients with higher miR-186-5p expression level had a better overall survival (OS) (Fig. 5C). BCa patients with higher mAPP expression level had a worse prognosis (Fig. 5D). In BCa tissues, the results of In Situ Hybridization (ISH) assay released that the expression of APP positively associated with the expression of circAPP (Fig. 5E). 


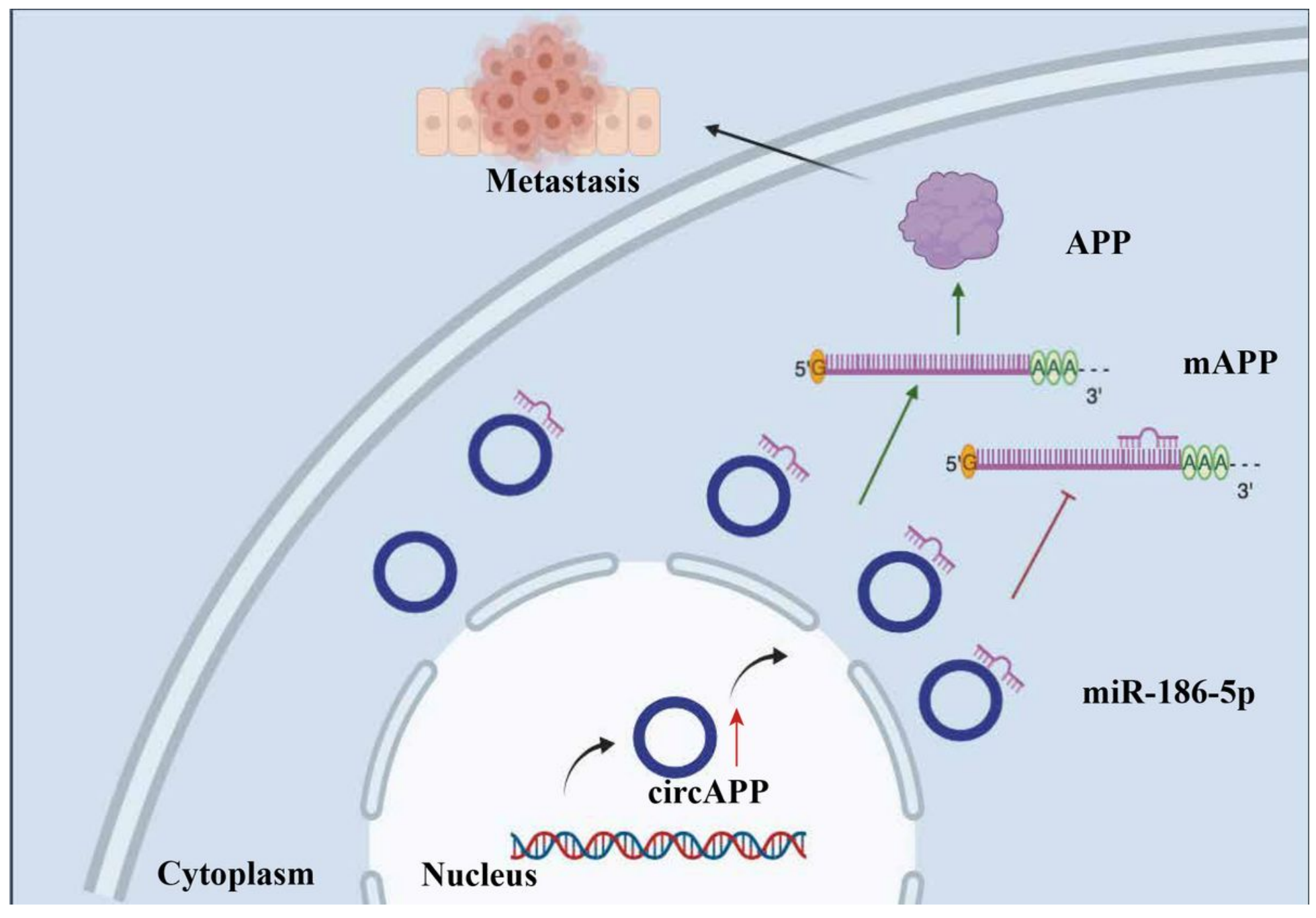

\section{Figure 6}

Importantly, circAPP could increase the expression of its host gene APP via competing endogenous miR186-5p. Therefore, circAPP may be a promising independent prognostic biomarker and potential target in BCa therapy (Fig. 6). 